\title{
Impact of ploidy level on the distribution of Pokey element insertions in the Daphnia pulex complex
}

\author{
Roland Vergilino ${ }^{1,2,3^{*}}$, Shannon HC Eagle ${ }^{2}$, Teresa J Crease ${ }^{2}$ and France Dufresne ${ }^{1,3}$
}

\begin{abstract}
Background: Transposable elements (TEs) play a major role in genome evolution. Their capacity to move and/or multiply in the genome of their host may have profound impacts on phenotypes and dramatic consequences on genome structure. The population dynamics and distribution of TEs are influenced by their mode of transposition, the availability of niches in host genomes, and host population dynamics. Theories predict an increase in the number of TE insertions following hybridization or polyploidization. Evolution of TEs in hybrids and polyploids has mostly been studied in plants; few studies have examined the impacts of hybridization and/or polyploidization on TEs in animals. Hybrids and polyploids have arisen multiple times in the Daphnia pulex complex and are thought to reproduce by obligate parthenogenesis. Our study examines the effects of ploidy level on polymorphism and number of Pokey element insertions in diploid and polyploid hybrid isolates from the Daphnia pulex complex.

Results: The polymorphism of Pokey insertion sites did not depend solely on either the ploidy level or the genetic background of their host; therefore, it may be the result of interactions between these parameters and other parameters such as Pokey activity, selection and/or drift. No significant effect of ploidy level was found on the number of Pokey insertions using TE display and qPCR. However, the load of Pokey insertion sites and the number of unique insertion sites were slightly (but not significantly) higher in polyploids than in diploids.

Conclusions: These results suggest a lack of increase in the number of Pokey insertions following polyploidization but higher availability of Pokey insertion sites in polyploids than in diploids. Compared to previous TE display and qPCR results, the load of Pokey insertions in hybrid diploids was higher than in non-hybrid sexual and asexual diploids, which suggests an increase in the density of Pokey insertions following hybridization.
\end{abstract}

Keywords: Daphnia pulex, Hybrids, Insertion site polymorphism, Load, Pokey, Polyploids, Transposable element

\section{Background}

Transposable elements (TEs) are genetic components that are able to move and multiply within and between genomes. They are found in the genomes of almost all living organisms [1], although there are exceptions in endosymbiont organisms [2]. There is large variation in the proportion of TEs across genomes [3]. TE populations are impacted by host population dynamics, such as effective population size, mode of reproduction, hybridization, and polyploidization [4-6]. A decrease in effective population size of the host [7] or an increase in its level of selfing

\footnotetext{
* Correspondence: rvergilino@gmail.com

'Département de Biologie, Chimie et Géographie, Université du Québec à Rimouski, Rimouski, Québec G5L 3A1, Canada

${ }^{2}$ Department of Integrative Biology, University of Guelph, Guelph, Ontario N1G 2W1, Canada

Full list of author information is available at the end of the article
}

[8] are expected to lead to an increase in the density of TE insertions. The mode of reproduction of the host also has a substantial impact on the dynamics and density of TE insertions in the genome [9-12]. For example, TEs may spread via recombination and out-crossing in sexual populations [11], whereas the spread of TEs among lineages is prevented in asexual populations except by horizontal transmission [13,14]. Empirical studies have tested and are generally in accordance with the theoretical prediction that the genomes of sexual organisms will contain a higher number of TE insertions compared to asexual ones [10,12-15]. Hybridization and polyploidization, which play a significant role in the diversification of plants and animals [16-18], might also have an impact on the load and insertion site polymorphism of TEs. Activation of TEs has been observed in hybrid genomes [19-22] (however, there are contradictory results in hybrid sunflowers [23]), and 
polyploidization may lead to an increase in the density of TE insertions [24,25], although there are contradictory results in allopolyploid plants [26]. Bursts of TE activity are thought to have a substantial impact on genome rearrangement [27] and may lead to phenotypic diversification in hybrids and polyploids [24,28]. Many studies have explored the effects of hybridization and polyploidization on TE dynamics in plants [22,23,26,28-34], but few studies have focused on these effects in animals, with the exception of studies in carp [35], Drosophila [36] and wallaby [20], all of which have been reviewed [37]. Studying the dynamics of TEs in hybrids and polyploids may provide insight on the evolution of their genomes and their propensity to adapt to various environments.

The Daphnia pulex (D. pulex) species complex has been intensively studied due to its dominance in freshwater habitats in North America and its variation in reproductive mode and ploidy level. Daphnia usually reproduce by cyclic parthenogenesis, which is clonal reproduction interrupted by bouts of sexual reproduction. However, some lineages reproduce by obligate parthenogenesis (i.e., without any sexual reproduction) [38-42]. The $D$. pulex complex includes numerous lineages that have been distinguished on the basis of morphological, ecological, and genetic data [43-47]. Analyses of mitochondrial DNA variation have revealed the presence of three major groups in this complex. The pulicaria group consists of five different lineages; North American D. pulicaria (with three sublineages, Eastern D. pulicaria, Western D. pulicaria and Polar D. pulicaria), D. pulex, $D$. melanica, $D$. middendorffiana sensu stricto, and $D$. arenata, an endemic species inhabiting Oregon ponds $[43,48]$. The tenebrosa group includes two lineages, European D. pulicaria and D. tenebrosa [43]. The third group includes European D. pulex. Mitochondrial lineages in the pulicaria group may have diverged during the Pleistocene (between 1.2 and 2.2 million years ago) [43,49,50] while the pulicaria and tenebrosa groups seem to have diverged during the Pliocene (around 3 million years ago) [43]. Relationships between lineages based on nuclear genes are less clear and may be confounded by incomplete lineage sorting and a highly reticulate history $[45,51,52]$. In North America, two lineages, D. pulex and D. pulicaria (considered to be ecological species), are dominant in freshwater habitats. They are morphologically similar but ecologically distinct [44], although they hybridize in nature [42,53-55]. D. pulex and F1 hybrids are usually found in fishless shallow ponds whereas D. pulicaria inhabits lakes. Variation in the Lactate dehydrogenase gene $(L d h)$ is diagnostic $[54,55] ; D$. pulex is fixed for the $\mathrm{S}$ allele whereas the F allele is fixed in D. pulicaria [55]. Diploid hybrids of these two lineages possess an SF genotype at the $L d h$ locus, always have $D$. pulex mitochondrial genomes, and have been found to reproduce by obligate parthenogenesis in nature [42,56], although laboratoryproduced hybrids may be able to reproduce by cyclical parthenogenesis [56,57]. It has been suggested that hybridization may play a role in the spread of meiosis suppressing genetic elements in the obligate parthenogenetic populations of $D$. pulex with SS Ldh genotypes via introgression [58,59].

Polyploidy has evolved repeatedly in the D. pulex complex [49,60-62] and shows a geographical pattern [49,62-66]. Polyploid populations are obligate parthenogens and are found at high latitudes and altitudes, and diploid populations (hybrid or not) are prevalent in temperate regions $[47,60,62]$. A polyphyletic assemblage of polyploids collectively known as $D$. middendorffiana (and which we term $D$. middendorffiana sensu lato in this study) is thought to have arisen from hybridization between $D$. pulex males and $D$. pulicaria females, or females of another species which no longer exists as a cyclic parthenogen $[49,60,61]$. Other polyploids are thought to have arisen from crosses between D. pulex females and D. pulicaria males and are encountered in the Northeast of Quebec and in Ontario (Canada) [45,67]. Moreover, D. tenebrosa, a circumarctic species [62], includes both diploids and polyploids [67], but the hybrid nature of the polyploids in this species is still unclear [45]. A study using microsatellite data, flow cytometry, and mitochondrial sequences has shown that most polyploids of the $D$. pulex complex are triploids, although some tetraploids have also been observed [67].

The $D$. pulex genome of one cyclically parthenogenetic isolate from Oregon has been sequenced [68], and numerous class II TEs have been identified in it [9]. Previous studies have reported that the class II TE load is lower in the genomes of obligate compared to cyclical parthenogenetic D. pulex lineages $[10,15]$, as theoretically predicted if sexual reproduction helps TEs to spread [11]. Pokey, a class II TE from the piggyBac superfamily, has been extensively studied in diploid populations of Daphnia. It inserts in the tandemly repeated rRNA genes [69] and in other parts of the genome [15,70]. Based on patterns of polymorphism in Pokey insertion sites observed among natural populations, previous studies have suggested that Pokey may be active in cyclically parthenogenetic populations of $D$. pulex but not in obligate parthenogens $[15,70]$. The diversity, and potentially the activity, of Pokey in rRNA genes is greatly influenced by recombination events, especially in hybrids [71]. The D. pulex complex and Pokey represent an interesting model to study the effect of hybridization and polyploidization on the evolution and dynamics of a class II TEs in natura.

The aim of this study is to compare the polymorphism of Pokey insertion sites between diploid and polyploid hybrid genomes in obligately parthenogenetic isolates of the D. pulex complex. If Pokey was not active during 
and following hybridization events, the similarity between Pokey insertion profiles should be congruent with host evolutionary relationships. To test this prediction, the polymorphism of Pokey insertion site profiles was compared with the ploidy level and the genetic similarity of the hosts determined using microsatellite multilocus genotypes. Moreover, we test the prediction that the number of Pokey per haploid genome (hereafter called density) is similar in polyploid and diploid hybrids using two complementary techniques, TE display and quantitative PCR (qPCR). TE display allows us to compare the diversity of Pokey insertion sites in polyploid and diploid isolates. This technique also provides an estimate of the number of Pokey insertion sites (Pokey load) but it cannot distinguish between homozygosity and heterozygosity at a particular site. Conversely, qPCR allows us to estimate the total number of Pokey insertions per haploid genome (Pokey density) regardless of location, including those that occur in rDNA, which appear as a single peak in a TE display analysis. A higher density of Pokey insertions per haploid genome in polyploids than in diploids may be evidence of an increase in Pokey activity after polyploidization.

\section{Methods}

\section{Daphnia samples}

In the laboratory, we established parthenogenetic lines of Daphnia (hereafter called isolates) from 27 individual obligately parthenogenetic females (14 diploid hybrids and 13 polyploid hybrids) sampled from ponds in North America between 2004 and 2008 (Additional file 1). Daphnia were sampled from ponds accessed via public roadsides or on private land with the permission of the land owner. No specific permissions are required to sample Daphnia as they are not endangered or protected species. The lines were cultured using standard techniques [72]. The isolates represent six mitochondrial lineages (D. pulex, Polar D. pulicaria, Western D. pulicaria, Eastern D. pulicaria, D. middendorffiana sensu stricto, and D. tenebrosa). Due to the geographical polyploidy pattern, all the polyploids come from two subarctic regions (Churchill, MB, Canada and Kuujjuarapik, QC, Canada), although the diploids come from both temperate and subarctic regions (Additional file 1). For each isolate, genomic DNA from 10 to 30 individuals, weighing approximately $100 \mathrm{mg}$ (wet weight), was extracted using the DNeasy Tissue kit (QIAGEN Inc., Mississauga, ON, Canada) according to the supplier's protocol. Origin of the putative parental species of each isolate (Additional file 1) was determined by combining information on morphology, haplotype of the mitochondrial ND5 gene, and genotype at the nuclear $L d h$ gene [45]. Ploidy levels were previously assessed using nine microsatellite loci and flow cytometry [45].

\section{TE display}

We used a PCR-based approach called TE display [73], which generates dominant AFLP-like markers, to test the effect of ploidy level on insertion site polymorphism, and on the load of Pokey insertions in the genomes of 14 diploid and 13 polyploid isolates. We followed a modified version of the TE display protocol of Valizadeh and Crease [15] that involves digestion of genomic DNA using the restriction enzyme BfaI followed by ligation of BfaI linkers and two rounds of PCR amplification using a Pokey-specific forward primer (Additional file 2). The ligated DNA was used as a template for a primary (preselective) PCR with the primer Pok6456F, located near the 3' end of Pokey, and the primer BfaI-R that anneals to the BfaI linker sequence followed by a secondary (selective) PCR using fluorescent labeled primer Pok6464F and the primer BfaI-R (Table 1). Our TE display protocol, unlike that of Valizadeh and Crease [15], used an annealing temperature of $50^{\circ} \mathrm{C}$ instead of $55^{\circ} \mathrm{C}$ for both the primary and secondary PCR. This allows amplification of Pokey insertions in Daphnia species with genomes that are divergent from $D$. pulex. Only fragments $\geq 160$ bp were included in our analyses and primary PCR were repeated three times in each individual followed by a secondary PCR on the product of each primary reaction to ensure that Pokey profiles were reproducible and to remove possible artefacts from our analysis (Additional file 2).

\section{Comparison of genetic distance based on Pokey profiles, microsatellites and the ND5 gene}

We used the results of TE display to generate a binary matrix of presence (1) or absence (0) of peaks, which represents the Pokey insertion profile (Additional file 3). We then generated a matrix of Jaccard distance estimates from the Pokey profiles. The Jaccard distance was chosen because it does not use shared absence of an allele as a shared characteristic [74]. A distance matrix was also calculated for each locus of the microsatellite dataset previously obtained for our isolates [45] using a modified version of the Bruvo distance [75], implemented in the PolySat package [76] using the R software [77]. The Bruvo distance allowed us to estimate relationships of mixed-ploidy level genotypes using co-dominant markers. The Bruvo distance takes into account stepwise mutation models between alleles. In the non-modified version (equation 2 in [75]), the algorithm adds "virtual allele" with an "infinite" value to lowest ploidy-level genotypes to compare them to the highest ploidy-level genotypes. This may lead to group artificially genotypes with the same ploidy level [78]. Thus, we used a modified version of the Bruvo distance (Bruvo2.distance implemented in PolySat set with the parameters add = TRUE and loss = TRUE) that allows genome "addition" and "loss", simulating gene addition by polyploidization but 
Table 1 TE display and qPCR primers and linkers used in this study

\begin{tabular}{|c|c|c|c|c|c|}
\hline Purpose & $\begin{array}{c}\text { Annealing } \\
\text { temperature }\end{array}$ & Primer name & Sequence (Dye) & $\begin{array}{c}\text { Percent amplification } \\
\text { efficiency }\end{array}$ & Amplicon size \\
\hline \multirow[t]{2}{*}{ Linkers for TED } & I & Bfal Linker F & 5'-TACTCAGGACTCAT & / & / \\
\hline & & Bfal Linker R & 5'-GACGATGAGTCCTGAG & & \\
\hline \multirow[t]{2}{*}{ Primary PCR for TED } & $50^{\circ} \mathrm{C} / 55^{\circ} \mathrm{C}$ & Pok6456F & 5'-GACAACGGTGGCCGAAACGCGG & / & / \\
\hline & & BfalR & 5'-GACGATGAGTCCTGAGTAG & & \\
\hline \multirow[t]{2}{*}{ Secondary PCR for TED } & $50^{\circ} \mathrm{C} / 55^{\circ} \mathrm{C}$ & Pok6464F & 5'-TGGCCAAAACACGGTTTGGCCG (HEX) & / & / \\
\hline & & BfalR & 5'-GACGATGAGTCCTGAGTAG & & \\
\hline \multirow[t]{2}{*}{$18 S$ genes for $\mathrm{qPCR}$} & $60^{\circ} \mathrm{C}$ & 18S1864F & 5'-CCGCGTGACAGTGAGCAATA & 0.9556 & 50 \\
\hline & & 18S1913R & 5'-CCCAGGACATCTAAGGGCATC & & \\
\hline \multirow[t]{2}{*}{285 genes for $q \mathrm{PCR}$} & $60^{\circ} \mathrm{C}$ & $28 S 3054 F$ & 5'-GGTAGCCAAATGCCTCGTCA & 0.9246 & 150 \\
\hline & & 28S3204R & 5'-GAGTCAAGCTCAACAGGGTCTTCTTTCCC & & \\
\hline \multirow[t]{2}{*}{ Total Pokey for qPCR } & $60^{\circ} \mathrm{C}$ & Pok6456F & 5'-GACAACGGTGGCCGAAACGCGG & 0.9136 & 122 \\
\hline & & Pok6578R & 5'-GATGGTCGGATTCGATTGAATGCTCG & & \\
\hline \multirow[t]{2}{*}{ Pokey in rDNA for qPCR } & $60^{\circ} \mathrm{C}$ & Pok6456F & 5'-GACAACGGTGGCCGAAACGCGG & 0.8957 & 192 \\
\hline & & $28 S 3104 R$ & 5'-GTTAATCCATTCGTGCGCG & & \\
\hline \multirow[t]{2}{*}{ Tif for $\mathrm{qPCR}$} & $60^{\circ} \mathrm{C}$ & TIF392F & 5'-GACATCATCCTGGTTGGCCT & 0.9493 & 50 \\
\hline & & TIF442R & 5'-AACGTCAGCCTTGGCATCTT & & \\
\hline \multirow[t]{2}{*}{ Gtp for qPCR } & $60^{\circ} \mathrm{C}$ & GTP385R & 5'-TATTCAGCATGGAGAGACGGC & 0.9369 & 50 \\
\hline & & GTP435R & 5'-GATGTCGACTGACGCTGGAA & & \\
\hline
\end{tabular}

also possible gene loss via diploidization. This modified version of the Bruvo distance does not lead to artificially grouping genotypes with the same ploidy level altogether. In addition, we generated a matrix of sequence divergence between ND5 sequences from previous studies by Vergilino et al. [45,67] (Table 1 for Genbank accession number) from our isolates using the maximum composite likelihood model implemented in MEGA5.1 [79].

To determine if the Pokey insertion sites profiles differed depending on the genetic background and ploidy level between isolates, a principal coordinate analysis (PCoA) [80] and a K-means cluster analysis were conducted to represent affinities between the different Pokey insertion profiles or multilocus microsatellite genotypes using $\mathrm{R}$ software version 2.15.2 [77,81]. Each PCoA was constructed using the pco module of the labdsv library in the R software on the Jaccard distance matrix for the Pokey profiles and the modified Bruvo distance matrix for the microsatellites after transforming these distance matrices in Euclidean distances [82]. The K-means analyses were conducted on binary matrices representing either the Pokey profiles or microsatellite genotypes (transformed into a binary matrix), and the number of clusters for each analysis was set using an iterative method, CascadeKM with the calinski criterion [83], implemented in the vegan package available with $\mathrm{R}$ software. We also performed a Mantel test according to Legendre and Legendre (section 10.5 in [74]) using the Pearson method with 10,000 replicates (package vegan in $\mathrm{R}$ software) to compare the Jaccard distance matrix based on Pokey insertion profiles with both the Bruvo distance matrix based on microsatellite data and the distance matrix based on ND5 mitochondrial haplotypes.

To test the hypothesis that the load of Pokey insertions increases with ploidy level, we compared the number of Pokey insertion sites estimated by TE display to the ploidy level after taking into account the heterozygosity of the isolates. Heterozygosity was weighted by ploidy level and was calculated from the variability of nine microsatellite loci [45]. Theoretically, polyploids may arise from independent hybridization events and those with different parental genomes may possess a higher diversity of TE insertion sites than polyploids with similar parental genomes due to increased probability of homozygosity of some TE insertions in the latter case. If we do not account for the different genomes that form polyploids, then we may overestimate the effect of ploidy level since the number of TE insertions could be more strongly correlated with heterozygosity level than ploidy level. Thus, we introduce a ploidy-weighted heterozygosity index $\left(H_{p l}\right)$ for a comparison between diploids and triploids, which takes into account the ploidy level of each genotype such that:

$$
H_{p l}=\frac{n_{3}+0.5 * n_{2}}{n_{L}}
$$

where $n_{L}$ is the total number of microsatellite loci analyzed (9), $n_{3}$ is the number of loci with 3 different alleles, 
and $n_{2}$ is the number of loci with only 2 different alleles. Genotypes that are homozygous for all microsatellite loci have an $H_{p l}$ of 0 , diploid isolates that are heterozygous for every locus and triploid isolates with two different alleles at every locus have an $H_{p l}$ of 0.5 , and triploid isolates that have three different alleles at every locus have an $H_{p l}$ of 1 . Therefore, triploids with low $H_{p l}$ values (under 0.5) can be compared to diploid hybrids. To disentangle the effect of adding different genomes from the effect of increased ploidy level, we performed an ANCOVA (Analysis of Covariance) using $\mathrm{R}$ software [77] with the number of Pokey insertions as the dependent variable and the ploidy level and $H_{p l}$ as the independent variables. The number of singletons (i.e., Pokey insertion sites encountered in only one isolate) between diploid and polyploid isolates was compared using a Fisher exact test performed on a $2 \times 2$ contingency table similar to the approach of Wright et al. [73].

Direct comparison of our results to those obtained on cyclic and obligate non-hybrid diploid populations previously studied by Valizadeh and Crease [15] was not possible as these authors used a higher annealing temperature $\left(55^{\circ} \mathrm{C}\right.$ instead of $\left.50^{\circ} \mathrm{C}\right)$. Therefore, we performed additional TE display assays on six diploid hybrid and six polyploid hybrid isolates using the $55^{\circ} \mathrm{C}$ annealing temperature of Valizadeh and Crease [15].

\section{qPCR assays}

Because TE display generates dominant markers, it provides more information about the polymorphism of Pokey insertion sites than their density within the genome. This difference may be significant especially if a significant proportion of Pokey insertions are homozygous, which may be possible in polyploids [24,25]. Therefore, to help resolve this problem, the number of Pokey insertions per haploid genome was estimated using qPCR. We performed qPCR assays on Pokey inserted in 28S rRNA genes (rPokey) and in the entire genome ( $\mathrm{t}$ Pokey) of 9 diploid and 10 polyploid isolates as described by Eagle and Crease [84]. We also estimated the number of $18 \mathrm{~S}$ and $28 \mathrm{~S}$ rRNA genes as the number of rPokey may be correlated to the number of rRNA genes $[84,85]$. Briefly, we used the $\Delta C_{\mathrm{T}}$ qPCR method as described in Eagle and Crease [84] (Additional file 2) to estimate the density of multicopy genes (18S, 28S, tPokey, rPokey) relative to two single-copy genes (Table 1); Tif, a transcription initiation factor and Gtp, a member of the RAB subfamily of small GTPases. Assuming that diploids have two copies and triploids have three copies of these two genes, these estimates correspond to the haploid number of multicopy genes in each genome. Reaction conditions were run in triplicate as described in Eagle and Crease [84] (Additional file 2). The mean haploid copy number, rounded to the nearest 0.5 for diploids and 0.34 for triploids, and standard deviations were calculated for each multicopy gene in each isolate. The number of Pokey insertions outside 28S rRNA genes per haploid genome (gPokey) was calculated as [tPokey number - rPokey number].

We used modules available in the $\mathrm{R}$ software package to perform correlation and regression analyses between the haploid number of 18S rRNA genes, 28S rRNA genes, rPokey, and gPokey in diploids and polyploids. Levene's tests (equality of variances) and Student's $t$-tests (equality of means) were used to test for possible significant differences in 18S, 28S, rPokey, and gPokey haploid numbers between diploids and polyploids. The sequential Bonferroni technique proposed by Rice [86] was used to adjust the significance level $(0.05)$ for the multiple Student's $t$-tests comparing $18 \mathrm{~S}$ and $28 \mathrm{~S}$ number within isolates.

Under the assumption that the same gPokey elements are amplified using qPCR and TE display techniques, we can estimate the average heterozygosity for these elements in diploids by using:

$$
H_{\text {Pokey }}=\frac{2\left(n_{T E D-1}-n_{g P o k e y}\right)}{n_{T E D-1}}
$$

where $n_{T E D-1}$ represents the number of different Pokey insertion sites estimated by TE display minus the peak representing rPokey, and $n_{\text {gPokey }}$ is the haploid number of gPokey estimated by qPCR. However, due to partial heterozygosity in triploids, we were not able to calculate their exact heterozygosity level. The ratio $\left(k^{*} n_{\text {gPokey }}\right) /$ $n_{T E D-1}$, where $k$ is the ploidy level of the isolate, allows us to evaluate if TE display and/or qPCR techniques underestimate or overestimate Pokey insertions. If every gPokey insertion is in a heterozygous state, this ratio will be 1 . If the ratio is below 1, qPCR underestimates or TE display overestimates the number of Pokey insertions. If all insertions are in a homozygous state, the ratio equals 2 for diploids and 3 for triploids. If the ratio is greater than 2 or 3 in diploids or triploids, respectively, qPCR overestimates or TE display underestimates the number of Pokey insertions.

\section{Results}

\section{Polymorphism of Pokey insertion site profiles}

Using the TE display technique, the average number of Pokey insertion sites in 14 diploid isolates was 16.64 $( \pm 4.94)$, with values from 6 to 26 , whereas the average number of Pokey insertion sites in 13 polyploid isolates was $19.00( \pm 4.36)$, with values from 12 to 27 . The two means are not significantly different (Student t-test, $t=-1.3105, d f=25, P=0.202$; Table 2; Additional file 1). Overall, 88 different Pokey insertion sites were detected (Additional file 3). Such polymorphism allowed us to 
Table 2 Summary of TE display and qPCR analyses of Pokey number in diploid and polyploid isolates in the Daphnia pulex complex

\begin{tabular}{|c|c|c|c|c|c|c|c|c|}
\hline \multirow[t]{2}{*}{ Isolates } & \multicolumn{2}{|c|}{ TE display } & \multicolumn{6}{|c|}{ qPCR } \\
\hline & Anneal at $50^{\circ} \mathrm{C}$ & Anneal at $55^{\circ} \mathrm{C}$ & $18 \mathrm{~S}$ genes & $28 \mathrm{~S}$ genes & Total Pokey & rDNA Pokey & $\begin{array}{l}\text { Genome Pokey } \\
\text { (Total-rDNA) }\end{array}$ & Tif:Gtp \\
\hline $\begin{array}{l}\text { Diploids with known } \\
\text { hybrid status }\end{array}$ & $19.09 \pm 3.99[11]$ & $13.83 \pm 4.22[6]$ & $293.25 \pm 111.19[8]$ & $486.25 \pm 201.36[8]$ & $17.81 \pm 4.35[8]$ & $5.19 \pm 5.03[8]$ & $12.63 \pm 5.04[8]$ & $0.91 \pm 0.06[8]$ \\
\hline $\begin{array}{l}\text { Polyploids with known } \\
\text { hybrid status }\end{array}$ & $21.50 \pm 3.54[10]$ & $16.33 \pm 1.75[6]$ & $214.57 \pm 62.34[7]$ & $346.67 \pm 113.86[7]$ & $15.57 \pm 3.43[7]$ & $3.10 \pm 1.07[7]$ & $12.48 \pm 3.20[7]$ & $0.92 \pm 0.07[7]$ \\
\hline Total diploids & $16.64 \pm 4.94[14]$ & $12.71 \pm 4.86[7]$ & $292.78 \pm 104.02[9]$ & $488.28 \pm 188.45[9]$ & $16.67 \pm 5.32[9]$ & $5.05 \pm 4.72[9]$ & $11.61 \pm 5.61[9]$ & $0.90 \pm 0.06[9]$ \\
\hline Total polyploids & $19.00 \pm 4.36[13]$ & $14.44 \pm 3.17[9]$ & $248.03 \pm 75.21[10]$ & $398.10 \pm 127.42[10]$ & $14.33 \pm 3.45[10]$ & $3.77 \pm 1.40[10]$ & $10.57 \pm 4.03[10]$ & $0.92 \pm 0.08[10]$ \\
\hline
\end{tabular}

Results from qPCR are average \pm standard deviation of Pokey inserts per haploid genome whereas results from TE display are for whole genomes. Numbers in brackets are the number of isolates tested. Tif:Gtp ratio is the number of Tif relative to the number of Gtp single copy reference genes. 
analyze the similarity between isolates based on these Pokey profiles and on microsatellite genotypes using PCoA. In addition, the Pokey profiles and the genetic similarity of the hosts based on microsatellite loci and mitochondrial haplotypes were compared using Mantel tests.

The first two axes of the PCoA accounted for 23.3\%, with $12.4 \%$ for axis 1 and $10.9 \%$ for axis 2, of the total variability in Pokey profiles (Figure 1A; Additional file 4A). For the host genetic backgrounds, the first two axes of the PCoA accounted for $24.0 \%$, with $16.0 \%$ for axis 1 and $8.0 \%$ for axis 2 , of the total microsatellite variability (Figure $1 \mathrm{~B}$ and Additional file 4B). Pokey profiles and their host microsatellite genotypes ordinate differently in each PCoA according to the two first axes (Figure 1). Although according to the PCoA on the host genetic background, axis 2 differentiates the hybrids with $D$. pulex mitochondria from the $D$. middendorffiana sensu lato (Figure 1B); it is axis 1 that differentiates these isolates according the PCoA from Pokey profiles. The second PCoA axis of the Pokey profiles does not show clear differentiation pattern according to the mitochondrial haplotype or the ploidy level (Figure 1A). The Kmeans analyses of both Pokey profiles and microsatellite genotypes separate the isolates into two clusters $(K=2$, Calinski criterion), but each cluster represents a different set of isolates in the two datasets (Figure 1). One cluster based on Pokey profiles contains only polyploid isolates from Kuujjuarappik (Quebec) and Churchill (Manitoba) possessing D. pulex (PX3-QC-1 and PX3QC-2), Eastern D. pulicaria (PC3-QC-1, PC3-QC-2 and PC3-QC-3) or Western D. pulicaria (PC3-MB-6) mitochondrial haplotypes. The second cluster based on Pokey profiles contains diploid hybrids with D. pulex mitochondrial haplotypes, D. tenebrosa isolates, and polyploids with Polar D. pulicaria (PC3-MB-4 and PC3MB-5) or D. middendorffiana sensu stricto (MI3-MB-2) mitochondrial haplotypes. In contrast, one cluster based on microsatellites contains all the $D$. tenebrosa isolates (both diploids and polyploids) while the other cluster contains all the other isolates. Despite these differences, the distance matrices of Pokey insertion site profiles and both microsatellite genotype and mitochondrial haplotype datasets are partially correlated according to Mantel tests (Mantel test; $r=0.3957, P=0.0001$ and $r=0.3047, P=0.003$, respectively). The third axis of the PCoA constructed from Pokey profiles (9.3\%, Additional file 4) may explain why this distance matrix is partially correlated with the distance matrix based on microsatellite genotypes. Pokey profiles from $D$. tenebrosa isolates ordinate together according to axis 3 in the PCoA of Pokey (Additional file 4A) as they ordinate altogether according to axis 1 in the PCoA of microsatellites (Additional file 4B).

As the purpose of our study was to test the effect of ploidy level and not the effect of hybridization, six isolates with $D$. tenebrosa mitochondrial haplotypes and unknown hybrid origin (TE2-MB-1, TE2-MB-2, TE2MB-3, TE3-MB-1, TE3-MB-2 and TE3-MB-3; Additional file 1) were excluded from the ANCOVA analysis. Excluding these isolates, 88 different Pokey insertion sites
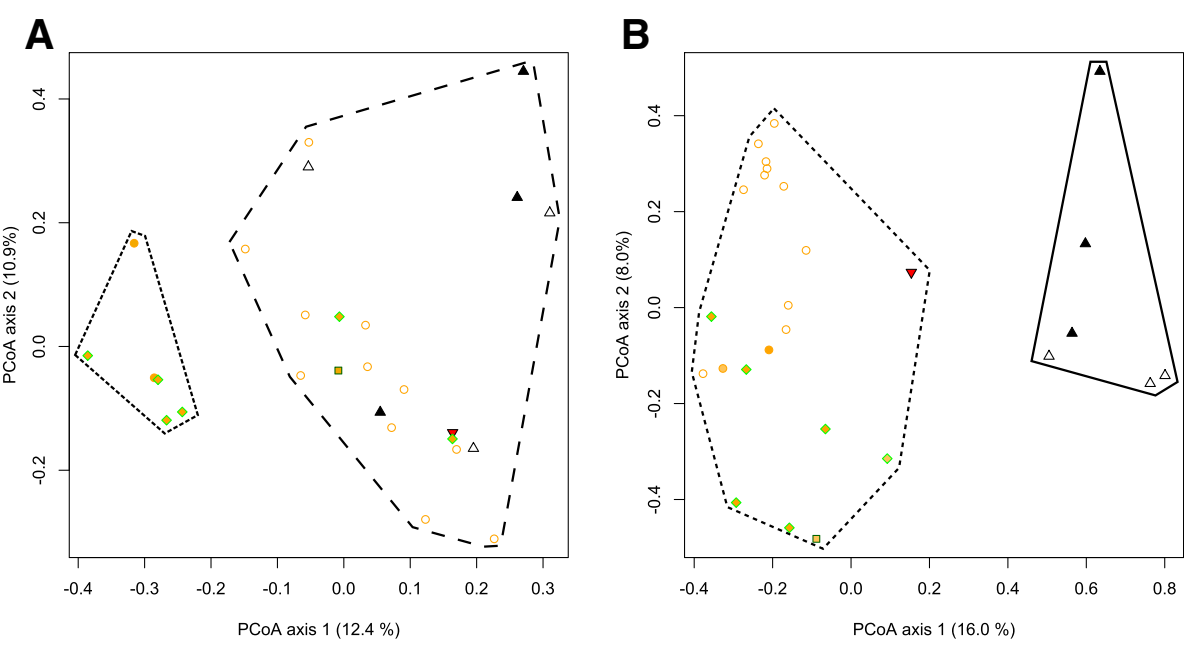

Figure 1 Principal Coordinate Analyses of Jaccard distance matrix of Pokey profiles and Bruvo distance matrix of microsatellite diversity in diploid and polyploid isolates of the Daphnia pulex complex. (A) Pokey profiles generated using TE display; (B) Microsatellite genotypes determined by Vergilino et al. [45]. The first two axes are represented in each graph. Empty symbols are diploids and solid symbols are polyploids. Empty orange circles: diploid hybrids with D. pulex mitochondrial haplotypes; solid orange circles: triploid hybrids with $D$. pulex mitochondrial haplotypes; solid square: D. middendorffiana sensu stricto; empty black triangles: diploid D. tenebrosa; solid black triangles: triploid $D$. tenebrosa; solid red triangle: introgressed D. tenebrosa with a D. pulex nuclear genome; solid green diamond filled with orange: triploid hybrids with D. pulicaria mitochondrial haplotypes. 
were detected using TE display (Additional file 3). The mode of reproduction is not a confounding effect in this analysis as both diploid and polyploid hybrids are obligate parthenogens. The mean number of Pokey insertion sites in diploid hybrid isolates is $18.09( \pm 3.99)$, with values from 13 to 26, whereas the mean number of Pokey insertion sites in polyploid isolates is $20.60( \pm 3.47)$, with values from 16 to 27 . These means are not significantly different (Student $t$-test, $t=-1.531, d f=19, P=0.1423$; Table 2; Additional file 1). Correlations between average number of Pokey insertion sites and the average heterozygosity weighted by host ploidy level $\left(H_{p l}\right)$ were positive but not significant (Table 3$). H_{p l}$, ploidy level and $H_{p l}$ :ploidy interaction had no significant effect on the number of Pokey insertion sites (ANCOVA, $F=2.132, d f=1, P=0.162$; $F=1.162, d f=1, P=0.296$ and $F=0.205, d f=1, P=0.657$, respectively; Figure 2; Additional file 5). The 11 diploid hybrid isolates displayed 65 of the 88 Pokey insertion sites, of which $8(12.3 \%)$ are singletons. The 10 polyploid hybrid isolates displayed 68 of the 88 Pokey insertion sites, of which 14 (20.6\%) are singletons. Twenty-one Pokey insertions sites were only sampled in polyploid hybrids while 18 were only sampled in diploid hybrids, and this difference is not statistically significant (Fisher exact test, $P=0.8559$ ). The difference in the number of singletons between diploid and polyploid hybrids is not statistically significant (Fisher exact test, $P=0.3579$ ). The number of Pokey insertion sites observed with TE display using an annealing temperature of $55^{\circ} \mathrm{C}$, as in Valizadeh and Crease [15], was lower than that observed using an annealing temperature of $50^{\circ} \mathrm{C}(12.71 \pm 4.86 \mathrm{vs} .16 .75 \pm 6.07$, paired Student's $t$-test, $t=3.9506, d f=6, P=0.008$ for diploids and $14.44 \pm 3.17$ vs. $18.78 \pm 4.86$; paired Student's $t$-test, $t=5.3072, \quad d f=8, \quad P=0.0007$ for polyploids; Additional file 1). The number of Pokey insertion sites was about two times lower with an annealing temperature of $55^{\circ} \mathrm{C}$ in some isolates. For example, 13 sites were detected using an annealing temperature of $50^{\circ} \mathrm{C}$ but only 6 were detected using $55^{\circ} \mathrm{C}$ in the diploid hybrid PX2-MB-1 (Additional file 1). Although we are aware that artefacts can be produced during the TE display process, these differences do not seem to be due to a higher frequency of artefacts at $50^{\circ} \mathrm{C}$ than in $55^{\circ} \mathrm{C}$ as most artifacts were encountered using both annealing temperatures and were excluded from analysis as indicated in the Methods section.

\section{qPCR analysis of rRNA gene and Pokey copy number}

Using the qPCR technique, we estimated the haploid number of $18 \mathrm{~S}$ genes, $28 \mathrm{~S}$ genes (Additional file 6), and

Table 3 Correlations between Pokey and rRNA gene number in diploid and polyploid Daphnia from North America

\begin{tabular}{|c|c|c|c|c|c|c|c|}
\hline Cytotypes & X-axis & Y-axis & Slope & $y$-intercept & $\mathrm{R}^{2}$ & P-value & Figure \\
\hline All diploids & $18 \mathrm{~S}$ & $28 \mathrm{~S}$ & 1.8038 & -39.8411 & 0.9901 & $1.76 \mathrm{e}-08^{*}$ & $A F 6^{8}$ \\
\hline All triploids & $18 \mathrm{~S}$ & $28 \mathrm{~S}$ & 1.6627 & -14.3154 & 0.9586 & $5.07 \mathrm{e}-07^{*}$ & AF6 \\
\hline \multirow[t]{4}{*}{ Diploid hybrids } & $H p I^{1}$ & Pokey $(\mathrm{TED})^{2}$ & 16.500 & 11.674 & 0.0634 & 0.4549 & 2 \\
\hline & $18 \mathrm{~S}$ & rPokey ${ }^{3}$ & -0.0146 & 9.4540 & 0.1035 & 0.4371 & AF7 \\
\hline & gPokey ${ }^{4}$ & rPokey & -06251 & 13.0792 & 0.3927 & 0.0964 & AF8 \\
\hline & Pokey-1 (TED) $)^{5}$ & Total gPokey ${ }^{6}$ & 1.5329 & -1.7681 & 0.4950 & 0.0515 & 3 \\
\hline \multirow[t]{2}{*}{ Diploid hybrids - PX2-MB-1 } & $18 \mathrm{~S}$ & rPokey & 0.0147 & -1.07571 & 0.3979 & 0.1287 & AF7 \\
\hline & gPokey & rPokey & -0.3350 & 8.0216 & 0.5605 & 0.0528 & AF8 \\
\hline \multirow[t]{3}{*}{ Diploid hybrids - (PX2-QC-9, PX2-MI-7) } & Pokey-1 (TED) & Total gPokey & 1.5741 & -2.7883 & 0.7383 & $0.0283^{*}$ & - \\
\hline & Pokey-1 (TED) & $H_{\text {Pokey }}^{7}$ & -0.0088 & 0.7623 & 0.0376 & 0.7130 & AF9 \\
\hline & Hpl & $H_{\text {pokey }}$ & -3.2220 & 1.8820 & 0.6592 & $0.0497^{*}$ & AF10 \\
\hline \multirow[t]{4}{*}{ Triploid hybrids } & $H p l$ & Pokey (TED) & 6.6490 & 17.534 & 0.0756 & 0.4418 & 2 \\
\hline & $18 \mathrm{~S}$ & rPokey & 0.0085 & 1.2673 & 0.2492 & 0.2541 & AF7 \\
\hline & gPokey & rPokey & 0.0201 & 2.8478 & 0.0036 & 0.8978 & AF8 \\
\hline & Pokey-1 (TED) & Total gPokey & -1.6300 & 1.0290 & 0.3342 & 0.1740 & 3 \\
\hline Triploid tenebrosa & Pokey-1 (TED) & Total gPokey & -0.1923 & 20.7692 & 0.4808 & 0.5122 & 3 \\
\hline
\end{tabular}

1. Denotes average heterozygosity estimated using nine microsatellite loci weighted by the ploidy level.

2. Denotes Pokey insertion site number estimated using TE display.

3. Denotes haploid Pokey number in $28 \mathrm{~S}$ genes amplified using $\mathrm{qPCR}$.

4. Denotes haploid Pokey number outside 285 genes amplified using qPCR.

5. Denotes Pokey insertion site number estimated using TE display minus the peak from elements in $28 \mathrm{~S}$ genes.

6. Denotes total Pokey number outside $28 \mathrm{~S}$ genes amplified using qPCR.

7. Denotes average heterozygosity of Pokey insertions outside $28 \mathrm{~S}$ genes amplified using qPCR.

8. AF refers to Additional Files.

${ }^{*} \mathrm{P}$ values are significant results. 


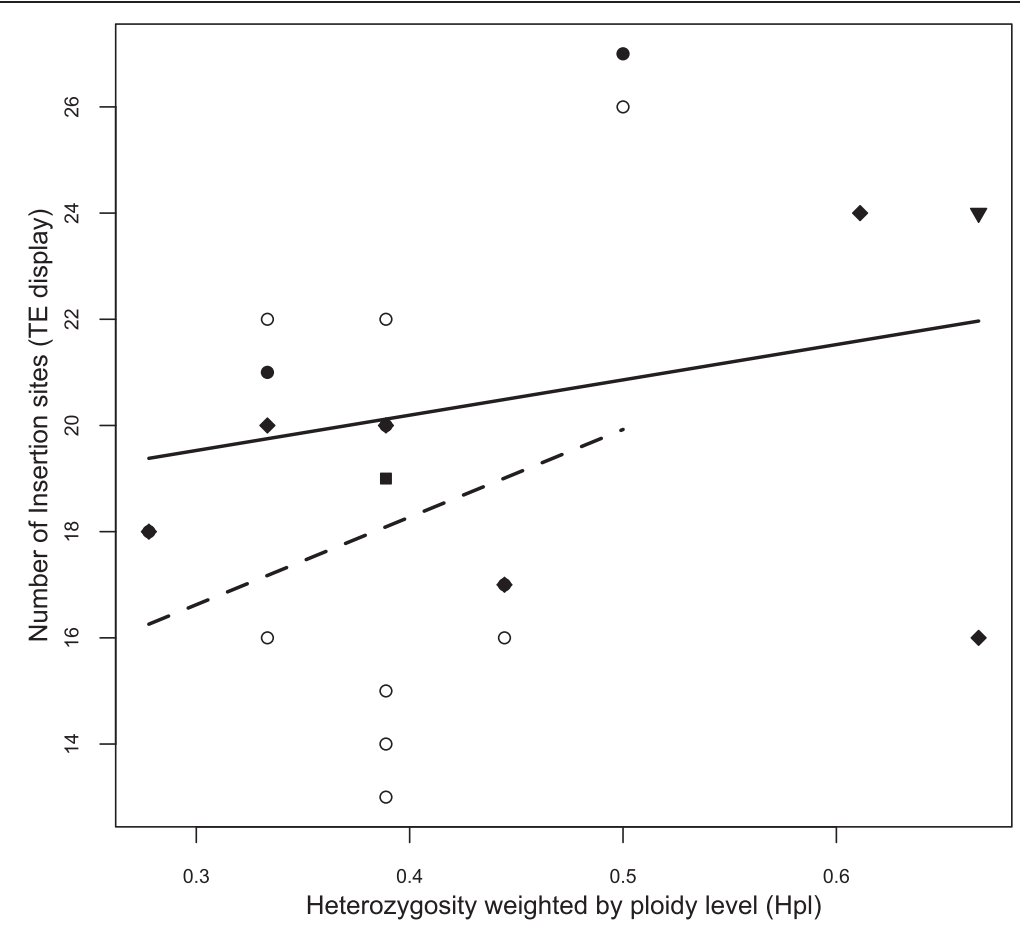

Figure 2 Relationship between Pokey insertion site number outside rDNA estimated using TE display and ploidy-weighted heterozygosity $\left(H_{p l}\right)$ in diploid and polyploid hybrids of the Daphnia pulex complex. Empty circles: diploid hybrids with $D$. pulex mitochondrial haplotypes; solid circles: triploid hybrids with D. pulex mitochondrial haplotypes; solid square: D. middendorffiana sensu stricto; solid triangle: introgressed D. tenebrosa with a D. pulex nuclear genome; solid diamond: triploid hybrids with D. pulicaria mitochondrial haplotypes. Dashed and solid lines are linear regressions estimated from the data.

Pokey inserted in $28 \mathrm{~S}$ genes (rPokey) and in the whole genome (tPokey) in 19 isolates including 9 diploids and 10 polyploids (Table 2 , Additional file 1 ). Using these estimates, we calculated the number of Pokey insertions outside 28S genes (gPokey $=\mathrm{tPokey}-\mathrm{rPokey}$ ). Under the assumption that Tif or Gtp reference genes were neither duplicated or lost in any of the isolates, we expect the Tif:Gtp ratio to be close to 1 , and this was the case with ratios ranging from 0.79 to 1.03 and a mean of 0.90 for diploids and 0.92 for polyploids (Table 2). It is unlikely that correlated losses or duplications of both genes would occur in multiple isolates and so we have assumed that diploids have two copies and triploids have three copies of each reference gene.

Both $18 \mathrm{~S}$ and $28 \mathrm{~S}$ genes showed a tendency towards a higher copy number per haploid genome in diploids than in polyploids $(292.78 \pm 111.19$ vs. $248.04 \pm 62.34$ for $18 \mathrm{~S}$ genes and $488.28 \pm 201.36 v s .398 .10 \pm 113.86$ for $28 \mathrm{~S}$ genes; Table 2), but differences between diploids and polyploids were not significant (Student's $t$-test; $t=1.0828$, $d f=17, P=0.2940$ for $18 \mathrm{~S}$ and Student's $t$-test; $t=1.2337$, $d f=17, P=0.2341$ for $28 \mathrm{~S})$. The estimates of $18 \mathrm{~S}$ and $28 \mathrm{~S}$ number within each isolate were significantly correlated (Additional file 6) but the slopes of the lines generated by plotting them relative to one another were above the expected value of 1.0 with values of 1.80 for diploid hybrids and 1.66 for polyploids (Table 3 ). It is possible that we overestimated the number of $28 \mathrm{~S}$ genes (Additional file 6 ) and so the number of $18 \mathrm{~S}$ genes was used as a proxy of rDNA copy number in all subsequent analyses.

Excluding isolates with unknown hybrid nature, the average haploid number of tPokey insertions was $17.81 \pm 4.35$ for diploid hybrids and $15.58 \pm 3.43$ for polyploid hybrids (Table 2) and the difference was not statistically significant (Student's $t$-test; $t=1.1092, d f=13, P=0.2947$ ).

The number of rPokey was higher in diploids than in polyploids (mean 5.19 vs. 3.10, respectively), but this difference was not significant (Student's $t$-test; $t=1.0735$, $d f=13, P=0.3026)$. Variation in the number of rPokey insertions was higher in diploid than in polyploid hybrids (SD 5.03 vs. 1.07) but the difference was not statistically significant (Levene's test, $W=2.0149, \quad P=0.1793$ ). No correlation was found between the number of rPokey and the number of $18 \mathrm{~S}$ genes for either ploidy level (Table 3; Additional file 7) even if the outlier PX2-MB-1, which possesses a high number of rPokey (16.5) and a low number of $18 \mathrm{~S}$ genes compared to other diploids (Table 3), was omitted from the analysis.

The mean number of gPokey was 12.63 in diploid hybrids and 12.48 in polyploid hybrids (Table 2) and the 
difference was not significant (Student's $t$-test; $t=0.0653$, $d f=13, P=0.9489)$. Variation in the number of gPokey insertions was higher in diploid than in polyploid hybrids (SD 5.04 vs. 3.20) but the difference was not statistically significant (Levene's test, $W=0.8830$, $P=0.3645)$.

No significant correlation was found between the number of gPokey and the number of rPokey in hybrids of either ploidy level (Table 3; Additional file 8). The negative (but not significant) relationship between $\mathrm{rPo}$ key and gPokey in diploids was partly due to the high number of rPokey in the isolate PX2-MB-1. However, the relationship was still negative and was nearly significant when this isolate was discarded from the analysis (Table 3; Additional file 8).

\section{Comparison of TE Display and qPCR}

The assumption that the gPokey elements amplified using qPCR and TE display are identical seems to be reasonable (Figure 3). Of all 19 isolates, only one (PX2MI-7) had a ratio $\left(k^{*} n_{g \text { Pokey }}\right) / \mathrm{n}_{\text {TED-1 }}$ below 1 which may indicate an overestimation of gPokey number using TE display compared to qPCR. One diploid isolate (PX2QC-2) had a ratio above 2 and a triploid isolate (MI3MB-2) had a ratio above 3 (Figure 3). This suggests that either the number of gPokey was overestimated by qPCR, underestimated by TE display, or both. The relationship between gPokey number based on qPCR and TE display is positive and significant in diploid hybrids (Table 3; Figure 3). This relationship was negative but not significant in triploid D. tenebrosa and in triploid hybrids (Table 3; Figure 3).

After excluding isolates outside the lower and upper limits of possible values of total gPokey insertions estimated with qPCR and TE display (values of the ratio $\left(k^{*} n_{\text {gPokey }}\right) / n_{\text {TED-1 }}$ between 1 and 2 for diploids and between 1 and 3 for triploids), the average heterozygosity across Pokey insertions loci among diploid hybrids is $59.85 \%$. The relationship between the heterozygosity of Pokey-inserted loci and the number of gPokey estimated using TE display is slightly negative but not significant (Table 3; Additional file 9). The slope of the relationship between the ploidyweighted heterozygosity using nine microsatellite loci and the average heterozygosity of Pokey-inserted loci is negative and is significant for diploid hybrids (Table 3, Additional file 10).

\section{Discussion}

\section{The polymorphism of Pokey insertion sites in Daphnia} isolates

The polymorphism of TE insertion sites may depend on multiple factors such as selective pressure, drift, recombination rate, ploidy level, genomic background (i.e., the parental origins of the hosts), geographic location, and the characteristics of the element(s) hosted in the genome [8,25,31,73,87-94]. If the diversity of Pokey insertion sites is due to the admixture of haploid genomes from different species with different architecture (that is nucleotide variation, number of repetitive genetic structures, etc.), the similarity of Pokey profiles is expected to mirror the genetic relationship of their hosts. According to the PCoA (Figure 1), the pairwise distance between Pokey profiles of the Daphnia isolates is not congruent with their pairwise genetic distance based on nine microsatellite loci if only the two first axes are taken into account. Similarities between the patterns produced by TE display and microsatellite analyses can only be revealed if the third axis of the PCoA is taken into account (Additional file 4). According to the K-means analysis, clusters based on similarity of Pokey profiles are not congruent with clusters based on microsatellite genotypes. Conversely, Mantel tests indicated that similarity between Pokey profiles is partially correlated with distance matrices constructed from microsatellite diversity and with mitochondrial haplotype diversity $(r=0.3957$ and $r=0.3047$, respectively).

The polymorphism of Pokey insertion sites in the isolates studied here imperfectly follows their evolutionary relationship with one another. This is concordant with previous results in which sequences from rPokey elements amplified from some of the isolates included in this study show a different reticulation history than the one described by microsatellite data [71]. For example, rPokey sequences from triploids PC3-QC-1 and PX3QC-1, whose Pokey profiles cluster together using the K-mean analysis (Figure 1A), have Pokey sequences that are similar (Figure two in [71]). Similarly, rPokey sequences from triploid isolates from Churchill (MI3-MB-2 and PC3MB-5), whose Pokey profiles cluster with D. tenebrosa and diploid hybrid isolates in the K-means analysis (Figure 1A), are recombinant and show signatures of hybridization between D. tenebrosa and D. pulex or D. pulicaria. However, there was no indication of hybridization based on the analysis of microsatellite data, which clustered all $D$. tenebrosa isolates with one another (Figure 1B). Weider et al. [66] hypothesized introgression between $D$. tenebrosa and D. pulex or D. pulicaria based on mitochondrial DNA and allozyme analyses. The polymorphism of Pokey profiles may then mirror hybridization or introgression events between these species that microsatellites do not display due to homoplasy or null alleles in the microsatellite dataset. In our study, all D. tenebrosa isolates may be of hybrid origins but can still ordinate separately in the PCoA and cluster together in a separate group using the K-means analysis due to the sharing of a specific allele belonging to the D. tenebrosa species. Alternatively, Pokey insertion profiles may not correspond to the genetic relationships of 


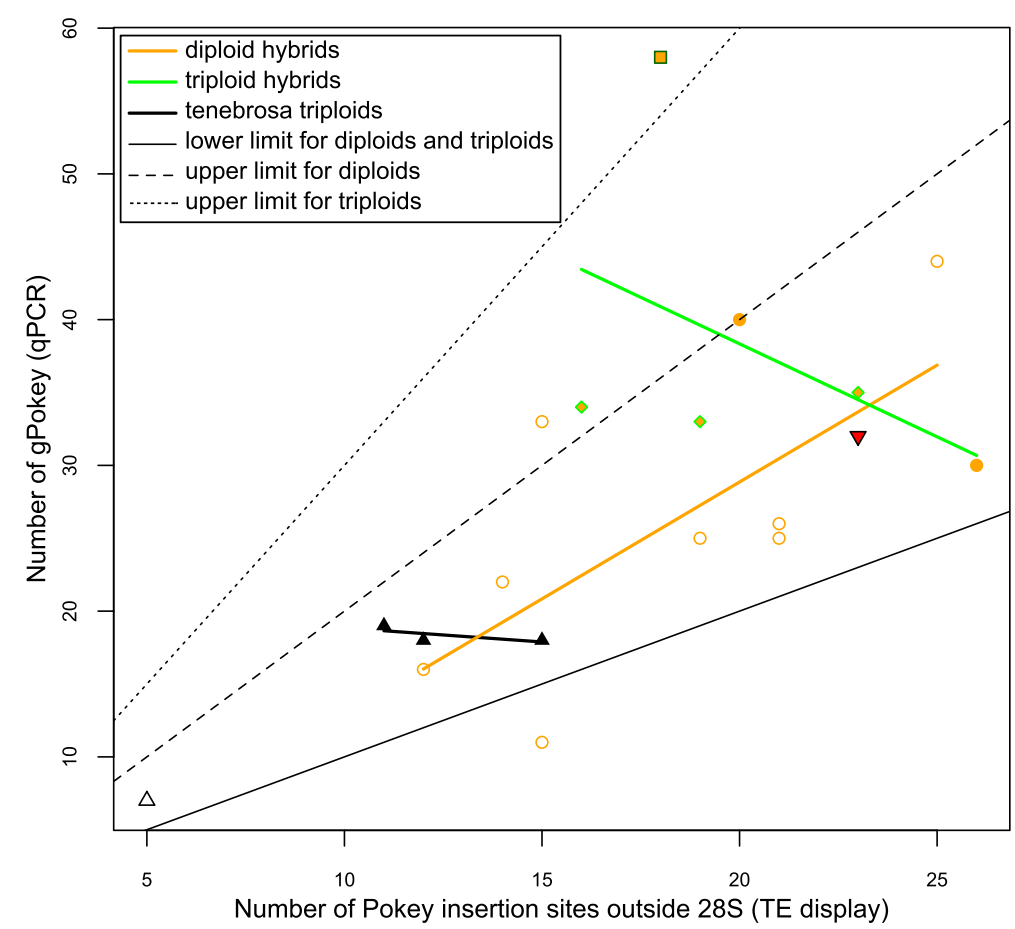

Figure 3 Relationship between the total number of Pokey insertions outside rDNA estimated using qPCR and TE display. Empty symbols are diploids and solid symbols are polyploids. Empty orange circles: diploid hybrids with $D$. pulex mitochondrial haplotypes; solid orange circles: triploid hybrids with D. pulex mitochondrial haplotypes; solid square: D. middendorffiana; empty black triangles: diploid D. tenebrosa; solid black triangles: triploid $D$. tenebrosa; solid red triangle: introgressed $D$. tenebrosa with a D. pulex nuclear genome; solid green diamond filled with orange: triploid hybrids with D. pulicaria mitochondrial haplotypes. Thin lines represent lower (slope $=1$ and $y$-intercept $=0$ ) and upper limits for diploids (slope $=2$ and $y$-intercept $=0$ ) and triploids (slope $=3$ and $y$-intercept $=0$ ) of Pokey insertion number (see Methods). Thick lines are linear regressions estimated from the data.

their host due to genomic rearrangements and random loss of copies in the course of evolution.

Patterns of Pokey insertion site polymorphism cannot be explained solely by ploidy level as K-means analyses show that individuals with different ploidy levels group in the same cluster whereas triploid individuals may belong to different clusters (Figure 1A). Valizadeh and Crease [15] did not find a relationship between the similarity of Pokey profiles and the mode of reproduction using a Neighbor-Joining tree of Pokey profiles from cyclic and obligate isolates of $D$. pulex. They concluded that the absence of a relationship was due to the multiple origins of obligate parthenogenetic lineages from multiple cyclical parthenogenetic populations. Similarly, the absence of a relationship between similarity of Pokey profiles and ploidy level is likely due to the multiple and independent origins of polyploid isolates.

Is Pokey load higher in Daphnia polyploids than diploids? Our study examined the load of Pokey insertions in relation to ploidy level in natural populations of Daphnia. Both our diploid and polyploid isolates are hybrids (with the exception of some D. tenebrosa isolates that were excluded from the analyses of load) and are obligate parthenogens. These characteristics allow us to test the effect of ploidy level on the load of a class II transposable element without the confounding effects of hybridization per se and of different modes of reproduction. No significant differences in the density of Pokey insertions using either qPCR (per haploid genome) or TE display were found between diploid and polyploid hybrids, suggesting that an increase in ploidy level does not lead to an increase of Pokey insertions in the long term. The isolates studied were sampled from natural populations and the age of these clones is unknown. Previous studies have suggested that obligately parthenogenetic populations of $D$. pulex originated some 150,000 years ago [93] and that some polyploids from the $D$. pulex complex were produced during the Pleistocene [49]. It is possible that an increase in Pokey insertions occurs shortly after polyploidization as predicted by several hypotheses $[25,28,94]$, but that genomic reorganization results in the loss of Pokey insertions with time. Loss of TE insertions following polyploidization (in both the short and long term) seems to be the rule rather the exception in most allopolyploid plants [26], regardless of whether the TEs are active. Loss of TE 
insertions is thought to be due to genome rearrangements via unequal and ectopic recombination events between TEs at non-homologous loci. Therefore, the non-significant difference between diploid and polyploid hybrids may be due to loss of Pokey following polyploidization. Conversely, the absence of statistical significance may be due to the substantial variability in the number of Pokey in both groups, which may be due to high variability of Pokey load in the parents. For example, Eagle and Crease [84] surveyed 69 non-hybrid isolates of D. pulex and D. pulicaria from 22 sampling sites and found that gPokey number can vary from 4 to 24. Thus, gPokey number in hybrids between these species will also vary according to the gPokey load in their ascendants. Alternatively, the presence or absence of active Pokey elements in parental species may influence the subsequent proliferation of Pokey in the hybrid offspring and increase the variability of Pokey insertion sites in hybrids. However, if Pokey is not active during apomixis, it cannot proliferate in obligately parthenogenetic hybrid lineages - except perhaps through ameiotic recombination events.

It has been suggested that Pokey is not active in nonhybrid obligately parthenogenetic isolates but may be active in cyclically parthenogenetic isolates of $D$. pulex $[15,70]$ and D. pulicaria [84]. Even so, it is possible that Pokey may be active in hybrids at least in the first generations after their formation due to the presence of active Pokey in their ascendants. Therefore, increases in the density of Pokey insertions may depend on the activity of Pokey and the effectiveness of regulation of Pokey in hybrid genomes [21,95-97]. Testing the activity of Pokey in diploid hybrids and performing additional studies on a larger number of hybrid and non-hybrid isolates will enhance our understanding of the dynamics and increase, if any, of Pokey elements in $D$. pulex $\times D$. pulicaria hybrids.

There is no difference between the load of Pokey insertion sites based on TE display in the genomes of polyploid hybrids $\left(20.60 \pm 3.47\right.$ at $50^{\circ} \mathrm{C}$ and $16.33 \pm 1.75$ at $\left.55^{\circ} \mathrm{C}\right)$ compared to diploid hybrids $\left(18.09 \pm 3.99\right.$ at $50^{\circ} \mathrm{C}$ and $13.83 \pm 4.22$ at $55^{\circ} \mathrm{C}$ ). Similarly, there is no difference in the density of gPokey per haploid genome between polyploid (12.48 \pm 3.20$)$ and diploid $(12.63 \pm 5.04)$ hybrids based on qPCR (Table 2). Conversely, the number of singletons (TE display at $50^{\circ} \mathrm{C}$ ) is slightly higher in polyploids $(20.6 \%)$ than in diploids $(12.3 \%)$. The relationship between Pokey number and heterozygosity also differs between the two groups. gPokey number (qPCR) increases with an increase in Pokey insertion sites (TE display) in diploids (Figure 3) but decreases in triploids (although not significantly). Moreover, in diploids, Pokey heterozygosity tends to decrease, though not significantly, as the number of different Pokey insertion sites increases (Table 3, Additional file 9). In contrast, polyploid heterozygosity increases (Table 3 ) and the number of total gPokey insertions (qPCR) decreases when Pokey insertion sites (TE display) increases (Table 3, Figure 3). Taken together, these results may reflect relaxed selection on insertions at some sites in polyploid compared to diploid hybrids, as suggested by the genomic niche redundancy hypothesis [24,25]. For example, if two functioning copies of a gene are necessary and sufficient for survival of the host, a third copy could become a potential genomic niche for TEs in triploid individuals. If so, then no TEs should be inserted in this gene in diploids but one gene copy could carry an insert in triploids without causing a decrease in host fitness. Alternatively, the difference in the number of singleton sites between the two ploidy levels may simply be a consequence of insertion site polymorphism contributed by the additional chromosome sets carried by polyploids.

\section{Pokey in rDNA}

The mean haploid number of $18 \mathrm{~S}$ is higher (although not significant) in diploid hybrid isolates than in polyploid hybrids, but when the haploid rDNA copy number is multiplied by the ploidy level, the average is equal between diploid (586.5) and polyploid (586.5) hybrids with D. pulex, D. pulicaria or D. middendorffiana mtDNA haplotypes. Previous studies have shown that polyploid plant species, such as natural and artificial allotetraploid populations of Tragopogon [98] and allotetraploid and allohexaploid grass species [99], may experience rDNA rearrangement, including loss of rRNA gene copies. As most organisms have many more rRNA genes than they require for survival [100], it is possible that a loss of copies in polyploids is not harmful. Indeed, it is possible that selection could actually favor the loss of copies if polyploidization initially results in high rDNA copy numbers that are somewhat deleterious. It is noteworthy that the average haploid $18 \mathrm{~S}$ number (293.3 with values from 131.5 to 451 ) in the diploid hybrid isolates is more than $30 \%$ higher than the average haploid $18 \mathrm{~S}$ number in the non-hybrid diploid isolates of D. pulex (221.0 with values from 94 to 489.5 ) and D. pulicaria (217.3 with values from 97 to 444) studied by Eagle and Crease [84] using the same qPCR protocol. This difference is not statistically significant (one-way ANOVA; $F=2.418, d f=2, P=0.0961)$. However, the number of Daphnia diploid hybrids tested here is low $(\mathrm{n}=8)$ comparing to D. pulicaria $(\mathrm{n}=37)$ and $D$. pulex $(\mathrm{n}=43)$ isolates tested in the study by Eagle and Crease [84]. There is a high level of variation within each group and it will be interesting to confirm this pattern after analysis in a larger sample of diploid hybrids and laboratoryproduced hybrids.

The number of $28 \mathrm{~S}$ genes with rPokey insertions accounts for an average of $1.93 \%$ of rDNA (with only one isolate, PX2-MB-1, above 5\%; Table 2; Additional file 1), which is consistent with the results of Eagle and Crease 
[84] who found rPokey insertions in approximately $1 \%$ of rDNA units in non-hybrid $D$. pulex and $D$. pulicaria isolates. Moreover, and still in accordance with Eagle and Crease [84], we did not find a correlation between the number of rPokey and rDNA in Daphnia hybrid isolates, including the polyploids in which rDNA copy number per haploid genome is lower. This is consistent with the hypothesis that Pokey is not highly active in the rDNA of these species, and its number does not increase with the number of rDNA units. Even so, selection is not so efficient that it eliminates deleterious elements present at low copy number in a highly repetitive gene family $[84,101,102]$.

\section{The effect of hybridization on Pokey load}

Valizadeh and Crease [15] found a significantly lower (one-way ANOVA; $F=67.65, d f=3, P<0.001$ ) average number of Pokey insertion sites in obligately and in cyclically parthenogenetic diploid isolates of $D$. pulex (3.27 $\pm 2.07, \mathrm{n}=22$ and $5.18 \pm 2.24, \mathrm{n}=22$ respectively) compared to our survey of 12 obligately parthenogenetic hybrid isolates using the same annealing temperature $\left(55^{\circ}\right.$ C) in TE display (13.83 \pm 4.22 for six diploid hybrid isolates and $16.33 \pm 1.75$ for six polyploid hybrid isolates; Table 2). In addition, the qPCR estimate of gPokey density is higher (although not statistically significant; oneway ANOVA; $F=2.549, d f=2, P=0.085)$ in our reduced data set of diploid hybrid isolates (12.63 \pm 5.04 for eight diploid hybrids) than in the diploid non-hybrid isolates analyzed by Eagle and Crease (9.6 for D. pulex, $\mathrm{n}=43$, and 9.5 for $D$. pulicaria, $\mathrm{n}=37$ ) [84]. Therefore, there seems to be an increase in the density of Pokey insertions in the genomes of hybrid Daphnia. This increase could occur either during the early generations after hybridization by bursts of Pokey activity or, if Pokey elements are still active in hybrids, over a long period through the slow accumulation of Pokey insertions within the genome. Bursts of TE activity in hybrids have been highlighted in numerous homoploid hybrid plants [22,29], fruit flies [19,21,36] and wallabies [20]. In Drosophila melanogaster and D. virilis, hybrid dysgenic crosses may lead to bursts in activity of various TEs [21,97] due to release from cytoplasmic repression $[37,95,96,103]$. Interestingly, there is a slight trend of decreasing Pokey site heterozygosity (Table 3; Additional file 10) and a trend of increasing Pokey insertions sites (Figure 2) as host average heterozygosity increases. These trends suggest there may be increased activity of Pokey in hybrids that have the most evolutionarily divergent parents. Alternatively, increased genome and cell size favored by natural selection in new and/or stressful habitats may lead to a slow increase in the number of TEs in the genome [104]. Genetic drift may also lead to a slow increase in the number of TEs as suspected in sunflowers
[104]. Three hybrid species of sunflower inhabiting harsh environments show genome size expansion due to proliferation of numerous class I TEs (retrotransposons) [34]. However, the proliferation of TEs is rare in contemporary natural sunflower hybrid populations and in artificial hybrid crosses [23,33], which suggest an increase of TEs after hybrid establishment via population processes such as genetic drift or natural selection. Selection in marginal habitats or drift following hybridization could also lead to an increase of TE density in hybrid Daphnia genomes.

\section{Conclusions}

Using TE display and qPCR, we were able to describe insertion site polymorphism and the load of Pokey elements in diploid and polyploid hybrid isolates of the $D$. pulex species complex. The polymorphism of Pokey insertion sites was not congruent with the evolutionary history and genetic relationships of their hosts. Diploid and polyploid hybrids did not differ significantly in the number of Pokey insertions, using either qPCR or TE display, as has been shown in studies comparing diploid and polyploid plants. The number of singletons estimated with TE display is slightly higher in polyploid than in diploid hybrids. Together, these results may reflect a higher number of sites available for Pokey insertions in polyploid than in diploid hybrids, or an increase in polymorphism due to the combination of genomes with Pokey at different insertion sites. Compared to previous studies on Pokey in the D. pulex complex, we found the density of Pokey insertions per haploid genome to be higher in obligately parthenogenetic hybrids (both diploids and polyploids) than in non-hybrid diploids (either cyclical or obligate parthenogens) leading to the conclusion that hybridization may lead to an overall increase in Pokey insertions. The estimation of polymorphism and TE load in laboratory-produced hybrids and the analysis of additional samples of hybrids will provide more insight into the population dynamics of TEs in diploid and polyploid hybrids of Daphnia.

\section{Additional files}

\footnotetext{
Additional file 1: Characteristics of the Daphnia isolates used in this study. Labels of the isolates are composites of their characteristics. The first two letters represent the mitochondrial haplotype followed by the ploidy level ( $2 x$ or $3 x$ ), a 2 -letter country or state/province code and the isolate number. Mitochondrial haplotypes are as follows: $\mathrm{EPC}=$ Eastern $D$. pulicaria, $\mathrm{WPC}=$ Western $D$. pulicaria, $\mathrm{PPC}=$ Polar D. pulicaria, PanPX $=$ Panarctic $D$. pulex, MIDD $=D$. middendorffiana sensu stricto, $\mathrm{TENE}=D$. tenebrosa). Ldh is the Lactate dehydrogenase genotype and indicates the hybrid nature of each isolate. $H_{p l}$ is the ploidy-weighted heterozygosity. rRNA gene and Pokey number were determined using TE display and $\mathrm{qPCR} ; 50^{\circ} \mathrm{C}$ and $55^{\circ} \mathrm{C}$ are the annealing temperatures used to generate the PCR amplicons in TE display. Total Pokey $=$ all Pokey elements in the genome. rDNA Pokey $=$ Pokey elements in $28 \mathrm{~S}$ rRNA genes. Genomic Pokey = total -rDNA elements.
} 
TG ratio is the number of Tif relative to the number of Gtp single copy reference genes.

Additional file 2: Supplementary material and methods describing TE display and qPCR protocols.

Additional file 3: TE display profiles of diploid and polyploid isolates in the $D$. pulex species complex. Sum of the amplification signals (peaks) are presented for each isolate.

Additional file 4: Three-dimensional representation of Principal Coordinate Analyses of Jaccard distance matrix of Pokey profiles and Bruvo distance matrix of microsatellite diversity in diploid and polyploid isolates of the Daphnia pulex complex. (A) Pokey profiles generated using TE display; (B) Microsatellite genotypes determined by Vergilino et al. [45]. The three first axes are represented. Empty symbols are diploids and solid symbols are polyploids. Empty orange circles: diploid hybrids with pulex mitochondrial haplotype, solid orange circles: triploid hybrids with D. pulex mitochondrial haplotype, solid square: D. middendorffiana sensu stricto, empty black triangles: diploid D. tenebrosa, solid black triangles: triploid $D$. tenebrosa, solid red triangle: introgressed $D$. tenebrosa with $D$. pulex nuclear genome, solid green diamond filled with orange: triploid hybrids with $D$. pulicaria mitochondrial haplotype; (C) and (D) are screeplots and represent the eigenvalues of the axes of Principal Coordinate Analysis (A) and (B), respectively.

Additional file 5: Results of the covariance analysis (ANCOVA) of TE display results from diploid and polyploid isolates in the Daphnia pulex complex. Ploidy level and ploidy-weighted heterozygosity $\left(H_{p i}\right)$ were used as the independent variables.

Additional file 6: Relationship between $18 \mathrm{~S}$ and $28 \mathrm{~S}$ gene number in diploid and polyploid isolates of the Daphnia pulex complex.

(A) Histograms of haploid number of $18 \mathrm{~S}$ and $28 \mathrm{~S}$ genes in each isolate. (B) Correlation between 185 and 285 gene number. Symbols represent mitochondrial haplotypes: circles for isolates with D. pulex mitochondria, diamonds for isolates with $D$. pulicaria mitochondria, squares for isolates with $D$. middendorffiana mitochondria, triangles for isolates with

$D$. tenebrosa mitochondria and inverted triangles for introgressed D. tenebrosa. Empty symbols represent putative diploids and solid ones indicate polyploids. Dashed and solid lines are linear regressions estimated from the data in diploids and polyploids, respectively. The dotted line was generated by plotting $18 \mathrm{~S}$ gene number on both axes.

Additional file 7: Correlation between haploid number of Pokey in rDNA (rPokey) and haploid $18 \mathrm{~S}$ gene number in diploid and triploid isolates of the $D$. pulex species complex. Empty circles represent diploid isolates and solid circles represent triploid isolates. Red empty circle represents the diploid isolate PX2-MB-1. Dashed and solid lines are linear regressions estimated from the data. The dashed lines with long strokes represent the linear regression following diploid hybrids without the isolate PX2-MB-1.

Additional file 8: Correlation between the haploid number of Pokey in rDNA (rPokey) and the haploid number of Pokey in other genomic locations (gPokey) in diploid and triploid isolates of the D. pulex species complex. Empty circles represent diploid isolates and solid circles represent triploid isolates. Red empty circle represents the diploid isolate PX2-MB-1. Dashed and solid lines are linear regressions estimated from the data. The dashed lines with long strokes represent the linear regression following diploid hybrids without the isolate PX2-MB-1.

Additional file 9: Correlation between average Pokey insertion site heterozygosity $\left(H_{\text {gPokey }}\right)$ and the number of Pokey insertions outside rDNA based on TE display analysis of diploid isolates. The dashed line represents the linear regression estimated from the data.

Additional file 10: Correlation between average Pokey insertion site heterozygosity $\left(H_{g \text { Pokey }}\right)$ and heterozygosity $\left(H_{p l}\right)$ of their diploid hosts based on microsatellite loci. The dashed line represents the linear regression estimated from the data.

\section{Abbreviations}

D. pulex: Daphnia pulex; gPokey: Genomic Pokey elements inserted outside rDNA; Ldh: Lactate dehydrogenase gene; PCoA: Principal coordinate analysis;
qPCR: Quantitative PCR; rPokey: Pokey elements inserted in rDNA; rDNA: ribosomal DNA; TE: Transposable element; TED: Transposable element display.

\section{Competing interests}

The authors declare that they have no competing interests.

\section{Authors' contributions}

RV designed the project and wrote the manuscript. RV and SHCE planned the analyses. RV and SHCE conducted the analyses. All authors analyzed the data and contributed to the writing and editing of the manuscript. All authors approved the final manuscript.

\section{Acknowledgments}

RV acknowledges a scholarship from Centre d'études Nordiques and a travel fellowship from UQAR. SHCE was supported by an Ontario Graduate Studies Science and Technology Scholarship. We thank the Genomics Facility at the University of Guelph for assistance with the qPCR. Comments from two anonymous reviewers greatly improved the earlier version of this manuscript.

\section{Funding}

This work was supported by Discovery Grants from the Natural Sciences and Engineering Research Council (NSERC) of Canada to FD and to TJC.

\section{Author details}

'Département de Biologie, Chimie et Géographie, Université du Québec à Rimouski, Rimouski, Québec G5L 3A1, Canada. ²Department of Integrative Biology, University of Guelph, Guelph, Ontario N1G 2W1, Canada. ${ }^{3}$ Centre d'Études Nordiques, Université Laval, Québec G1V OA6, Canada.

Received: 10 September 2013 Accepted: 20 November 2013 Published: 2 January 2014

\section{References}

1. Feschotte C, Pritham EJ: DNA transposons and the evolution of eukaryotic genomes. Ann Rev Gen 2007, 41:331-368.

2. Shigenobu S, Watanabe H, Hattori M, Sakaki Y, Ishikawa H: Genome sequence of the endocellular bacterial symbiont of aphids Buchnera sp. APS. Nature 2000, 407:81-86.

3. Pidpala O, Yatsishina A, Lukash L: Human mobile genetic elements: structure, distribution and functional role. Cytol Genet 2008, 42:420-430.

4. Charlesworth B, Charlesworth D: The population dynamics of transposable elements. Gen Res 1983, 42:1-27.

5. Charlesworth D, Charlesworth B: Transposable elements in inbreeding and outbreeding populations. Genetics 1995, 140:415-417.

6. Charlesworth D, Wright SI: Breeding systems and genome evolution Curr Opin Genetics Dev 2001, 11:685-690.

7. Brookfield J, Badge R: Population genetics models of transposable elements. Genetica 1997, 100:281-294.

8. Wright S, Schoen D: Transposon dynamics and the breeding system. Genetica 1999, 107:139-148.

9. Schaack S, Choi E, Lynch M, Pritham E: DNA transposons and the role of recombination in mutation accumulation in Daphnia pulex. Genome Biol 2010, 11:R46.

10. Schaack S, Pritham EJ, Wolf A, Lynch M: DNA transposon dynamics in populations of Daphnia pulex with and without sex. Proc R S B 2010, 277:2381-2387.

11. Wright S, Finnegan D: Genome evolution: sex and the transposable element. Current Biol 2001, 11:R296-R299.

12. Zeyl C, Bell G, Green DM: Sex and the spread of retrotransposon Ty3 in experimental populations of Saccharomyces cerevisiae. Genetics 1996, 143:1567-1577.

13. Arkhipova I, Meselson M: Transposable elements in sexual and ancient asexual taxa. Proc Natl Acad Sci USA 2000, 97:14473-14477.

14. Gladyshev EA, Meselson M, Arkhipova IR: Massive horizontal gene transfer in bdelloid rotifers. Science 2008, 320:1210-1213.

15. Valizadeh $P, C$ Crease $T$ : the association between breeding system and transposable element dynamics in Daphnia pulex. J Mol Evol 2008, 66:643-654.

16. Mable BK: 'Why polyploidy is rarer in animals than in plants': myths and mechanisms. Biol J Linnean Soc 2004, 82:453-466. 
17. Otto SP: The evolutionary consequences of polyploidy. Cell 2007, 131:452-462.

18. Seehausen O: Hybridization and adaptive radiation. Trends Ecol Evol 2004 19:198-207.

19. Fontdevila A: Hybrid genome evolution by transposition. Cytogenet Genome Res 2005, 110:49-55.

20. O'Neill RJW, O'Neill MJ, Graves JAM: Undermethylation associated with retroelement activation and chromosome remodelling in an interspecific mammalian hybrid. Nature 1998, 393:68-72.

21. Petrov DA, Schutzman JL, Hartl DL, Lozovskaya ER: Diverse transposable elements are mobilized in hybrid dysgenesis in Drosophila virilis. Proc Natl Acad Sci USA 1995, 92:8050-8054

22. Shan X, Liu Z, Dong Z, Wang Y, Chen Y, Lin X, Long L, Han F, Dong Y, Liu B: Mobilization of the active MITE transposons mPing and Pong in rice by introgression from wild rice (Zizania latifolia Griseb.). Mol Biol Evol 2005, 22:976-990.

23. Ungerer MC, Kawakami T: Transcriptional dynamics of LTR retrotransposons in early generation and ancient sunflower hybrids. Genome Biol Evol 2013, 5:329-337.

24. Comai L: Genetic and epigenetic interactions in allopolyploid plants. Plant Mol Biol 2000, 43:387-399.

25. Matzke MA, Matzke AJM: Polyploidy and transposons. Trends Ecol Evol $1998,13: 241-251$.

26. Parisod C, Alix K, Just J, Petit M, Sarilar V, Mhiri C, Ainouche M, Chalhoub B, Grandbastien MA: Impact of transposable elements on the organization and function of allopolyploid genomes. New Phytol 2010, 186:37-45.

27. McClintock $B$ : The significance of responses of the genome to challenge. Science 1984, 226:792-801.

28. Madlung A, Masuelli RW, Watson B, Reynolds SH, Davison J, Comai L: Remodeling of DNA methylation and phenotypic and transcriptional changes in synthetic arabidopsis allotetraploids. Plant Physiol 2002, 129:733-746.

29. Ainouche M, Fortune P, Salmon A, Parisod C, Grandbastien MA, Fukunaga K, Ricou M, Misset MT: Hybridization, polyploidy and invasion: lessons from Spartina (Poaceae). Biol Invasions 2009, 11:1159-1173.

30. de Araujo PG, Rossi M, de Jesus EM, Saccaro NL, Kajihara D, Massa R, de Felix JM, Drummond RD, Falco MC, Chabregas SM, Ulian EC, Menossi M, Van Sluys MA: Transcriptionally active transposable elements in recent hybrid sugarcane. Plant J 2005, 44:707-717.

31. Hanson RE, Islam-Faridi MN, Crane CF, Zwick MS, Czeschin DG, Wendel JF, McKnight TD, Price HJ, Stelly DM: Ty1-copia-retrotransposon behavior in a polyploid cotton. Chromosome Res 2000, 8:73-76.

32. Kashkush K, Feldman M, Levy AA: Gene loss, silencing and activation in a newly synthesized wheat allotetraploid. Genetics 2002, 160:1651-1659.

33. Kawakami T, Dhakal P, Katterhenry AN, Heatherington CA, Ungerer MC: Transposable element proliferation and genome expansion are rare in contemporary sunflower hybrid populations despite widespread transcriptional activity of LTR retrotransposons. Genome Biol Evol 2011, 3:156.

34. Ungerer MC, Strakosh SC, Zhen Y: Genome expansion in three hybrid sunflower species is associated with retrotransposon proliferation. Current Biol 2006, 16:R872.

35. Dong L, Cuiping Y, Shaojun L, Liangguo L, Wei D, Song C, Jinpeng Y, Yun L: Characterization of a novel Tc1-like transposon from bream (Cyprinidae, Megalobrama) and its genetic variation in the polyploidy progeny of bream-red crucian carp crosses. J Mol Evol 2009, 69:395-403.

36. Labrador $M$, Fontdevila A: High transposition rates of Osvaldo, a new Drosophila buzzatii retrotransposon. Mol Gen Genet 1994, 245:661-674.

37. Arkhipova IR, Rodriguez F: Genetic and epigenetic changes involving (Retro) transposons in animal hybrids and polyploids. Cytogenet Genome Res 2013, 140:295-311.

38. Hebert PDN: Obligate asexuality in Daphnia. Am Nat 1981, 117:784-789.

39. Hebert PDN, Crease T: Clonal diversity in populations of Daphnia pulex reproducing by obligate parthenogenesis. Heredity 1983, 51:353-369.

40. Innes DJ, Schwartz SS, Hebert PDN: Genotypic diversity and variation in mode of reproduction among populations in the Daphnia pulex group. Heredity 1986, 57:345-355.

41. Innes DJ, Fox CJ, Winsor GL: Avoiding the cost of males in obligately asexual Daphnia pulex (Leydig). Proc R S Series B 2000, 267:991-997.

42. Hebert $P$, Finston T: Macrogeographic patterns of breeding system diversity in the Daphnia pulex group from the United States and Mexico. Heredity 2001, 87:153-161.
43. Colbourne JK, Crease TJ, Weider LJ, Hebert PDN, Dufresne F, Hobaek A Phylogenetics and evolution of a circumarctic species complex (Cladocera: Daphnia pulex). Biol J Linnean Soc 1998, 65:347-365.

44. Hebert PDN: The Daphnia of North America: An Illustrated Fauna. Guelph, Ontario: University of Guelph; 1995.

45. Vergilino R, Markova S, Ventura M, Manca M, Dufresne F: Reticulate evolution of the Daphnia pulex complex as revealed by nuclear markers. Mol Ecol 2011, 20:1191-1207.

46. Adamowicz SJ, Gregory TR, Marinone MC, Hebert PDN: New insights into the distribution of polyploid Daphnia: the Holarctic revisited and Argentina explored. Mol Ecol 2002, 11:1209-1217.

47. Aguilera X, Mergeay J, Wollebrants A, Declerck S, de Meester L: Asexuality and polyploidy in Daphnia from the tropical Andes. Limnol Oceanogr 2007, 52:2079-2088.

48. Colbourne JK, Hebert PDN: The systematics of North American Daphnia (Crustacea: Anomopoda): a molecular phylogenetic approach. Philos Trans R Soc London Series B 1996, 351:349-360.

49. Dufresne F, Hebert PDN: Pleistocene glaciations and polyphyletic origins of polyploidy in an arctic cladoceran. Proc R S Series B 1997 264:201-206.

50. Weider $L$, Hobaek A: Glacial refugia, haplotype distributions, and clonal richness of the Daphnia pulex complex in arctic Canada. Mol Ecol 2003, 12:463-473.

51. Crease TJ, Floyd R, Cristescu MA, Innes D: Evolutionary factors affecting Lactate dehydrogenase $A$ and $B$ variation in the Daphnia pulex species complex. BMC Evol Biol 2011, 11:212.

52. Cristescu ME, Constantin A, Bock DG, Cáceres CE, Crease TJ: Speciation with gene flow and the genetics of habitat transitions. Mol Ecol 2012, 21:1411-1422

53. Crease TJ, Stanton DJ, Hebert PDN: Polyphyletic origins of asexuality in Daphnia pulex. II. Mitochondrial-DNA variation. Evolution 1989, 43:1016-1026.

54. Hebert PDN, Schwartz SS, Ward RD, Finston TL: Macrogeographic patterns of breeding system diversity in the Daphnia pulex group. I. Breeding systems of Canadian populations. Heredity 1993, 70:148-161

55. Hebert PDN, Beaton MJ, Schwartz SS, Stanton DJ: Polyphyletic origins of asexuality in Daphnia pulex. I. Breeding-system variation and levels of clonal diversity. Evolution 1989, 43:1004-1015.

56. Innes DJ, Hebert PDN: The origin and genetic basis of obligate parthenogenesis in Daphnia pulex. Evolution 1988, 42:1024-1035.

57. Heier CR, Dudycha JL: Ecological speciation in a cyclic parthenogen: sexual capability of experimental hybrids between Daphnia pulex and Daphnia pulicaria. Limnol Oceanogr 2009, 54:492-502.

58. Tucker AE, Ackerman MS, Eads BD, Xu S, Lynch M: Population-genomic insights into the evolutionary origin and fate of obligately asexual Daphnia pulex. Proc Natl Acad Sci USA 2013, 110:15740-15745.

59. Xu S, Innes DJ, Lynch M, Cristescu ME: The role of hybridization in the origin and spread of asexuality in Daphnia. Mol Ecol 2013, 22:4549-4561.

60. Beaton MJ, Hebert PDN: Geographical parthenogenesis and polyploidy in Daphnia pulex. Am Nat 1988, 132:837-845.

61. Dufresne F, Hebert PDN: Hybridization and origins of polyploidy. Proc $R S$ Series B 1994, 258:141-146.

62. Dufresne F, Hebert PDN: Polyploidy and clonal diversity in an arctic cladoceran. Heredity 1995, 75:45-53.

63. Hobaek A, Weider $\sqcup$, Wolf HG: Ecological genetics of Norwegian Daphnia. III. Clonal richness in an Arctic apomictic complex. Heredity 1993, 71:323-330.

64. Ward RD, Bickerton MA, Finston T, Hebert PDN: Geographical cline in breeding systems and ploidy levels in European populations of Daphnia pulex. Heredity 1994, 73:532-543.

65. Weider LJ, Hobaek A, Colbourne JK, Crease TJ, Dufresne F, Hebert PDN: Holarctic phylogeography of an asexual species complex I. Mitochondrial DNA variation in arctic Daphnia. Evolution 1999, 53:777-792.

66. Weider LJ, Hobaek A, Hebert PDN, Crease TJ: Holarctic phylogeography of an asexual species complex - II. Allozymic variation and clonal structure in Arctic Daphnia. Mol Ecol 1999, 8:1-13.

67. Vergilino R, Belzile C, Dufresne F: Genome size evolution and polyploidy in the Daphnia pulex complex (Cladocera: Daphniidae). Biol J Linnean Soc 2009, 97:68-79.

68. Colbourne JK, Pfrender ME, Gilbert D, Thomas WK, Tucker A, Oakley TH, Tokishita S, Aerts A, Arnold GJ, Basu MK, Bauer DJ, Cáceres CE, Carmel L, Casola C, Choi JH, Detter JC, Dong Q, Dusheyko S, Eads BD, Fröhlich T, Geiler-Samerotte KA, Gerlach D, Hatcher P, Jogdeo S, Krijgsveld J, 
Kriventseva EV, Kültz D, Laforsch C, Lindquist E, Lopez J, et al: The ecoresponsive genome of Daphnia pulex. Science 2011, 331:555-561.

69. Penton EH, Sullender BW, Crease TJ: Pokey, a new DNA transposon in Daphnia (Cladocera: Crustacea). J Mol Evol 2002, 55:664-673.

70. Sullender BW, Crease TJ: The behavior of a Daphnia pulex transposable element in cyclically and obligately parthenogenetic populations. J Mol Evol 2001, 53:63-69.

71. Vergilino R, Elliott TA, Desjardins-Proulx P, Crease TJ, Dufresne F: Evolution of a transposon in Daphnia hybrid genomes. Mob DNA 2013, 4:7.

72. Hebert PDN, Crease TJ: Clonal coexistence in Daphnia pulex (Leydig): another planktonic paradox. Science 1980, 207:1363-1365.

73. Wright SI, Le QH, Schoen DJ, Bureau TE: Population dynamics of an Ac-like transposable element in self- and cross-pollinating Arabidopsis. Genetics 2001, 158:1279-1288.

74. Legendre P, Legendre L: Numerical Ecology. 2nd English edn. Amsterdam: Elsevier Science; 1998.

75. Bruvo R, Michiels NK, D'Souza TG, Schulenburg H: A simple method for the calculation of microsatellite genotype distances irrespective of ploidy level. Mol Ecol 2004, 13:2101-2106.

76. Clark LV, Jasieniuk M: POLYSAT: an R package for polyploid microsatellite analysis. Mol Ecol Resouces 2011, 11:562-566.

77. R Development Core Team: R: A Language and Environment for Statistical Computing, Vienna: R: Foundation for Statistical Computing. 2004. http://www.R-project.org.

78. Dufresne F, Stift M, Vergilino R, Mable BK: Recent progress and challenges in population genetics of polyploid organisms: an overview of current state-of-the-art molecular and statistical tools. Mol Ecol 2014, 23:40-69.

79. Tamura K, Peterson D, Peterson N, Stecher G, Nei M, Kumar S: MEGA5: Molecular Evolutionary Genetics Analysis using maximum likelihood, evolutionary distance, and maximum parsimony methods. Mol Biol Evol 2011, 28:2731-2739.

80. Gower JC: Some distance properties of latent root and vector methods used in multivariate analysis. Biometrika 1966, 53:325-338.

81. Ihaka R, Gentleman R: R: a language for data analysis and graphics. J Comp Graph Stat 1996, 5:299-314.

82. Jaccard P: Nouvelles recherches sur la distribution florale. Bull de la société Vaudoise des Sci Nat 1908, 44:223-270.

83. Calinski T, Harabasz J: A dendrite method for cluster analysis. Commun Stat Theor M 1974, 3:1-27.

84. Eagle SHC, Crease TJ: Copy number variation of ribosomal DNA and Pokey transposons in natural populations of Daphnia. Mob DNA 2012, 3:4.

85. Eagle SHC: Copy Number Variation of Ribosomal RNA Genes and the Pokey DNA Transposon in the Daphnia pulex Species Complex. Guelph, Ontario: University of Guelph; 2013.

86. Rice WR: Analyzing tables of statistical tests. Evolution 1989, 43:223-225.

87. Bartolomé C, Maside X, Charlesworth B: On the abundance and distribution of transposable elements in the genome of Drosophila melanogaster. Mol Biol Evol 2002, 19:926-937.

88. Charlesworth B, Langley CH, Sniegowski PD: Transposable element distributions in Drosophila. Genetics 1997, 147:1993-1995.

89. Dolgin ES, Charlesworth $\mathrm{B}$ : The effects of recombination rate on the distribution and abundance of transposable elements. Genetics 2008, 178:2169-2177.

90. Duret L, Marais G, Biemont C: Transposons but not retrotransposons are located preferentially in regions of high recombination rate in Caenorhabditis elegans. Genetics 2000, 156:1661-1669.

91. Rizzon C, Marais G, Gouy M, Biemont C: Recombination rate and the distribution of transposable elements in the Drosophila melanogaster genome. Genome Res 2002, 12:400-407

92. Wright $\mathrm{SI}$, Agrawal N, Bureau TE: Effects of recombination rate and gene density on transposable element distributions in Arabidopsis thaliana. Genome Res 2003, 13:1897-1903.

93. Paland S, Lynch M: Transitions to asexuality result in excess amino acid substitutions. Science 2006, 311:990-992.

94. Wendel JF: Genome evolution in polyploids. Plant Mol Biol 2000, 42:225-249.

95. Blumenstiel JP, Hartl DL: Evidence for maternally transmitted small interfering RNA in the repression of transposition in Drosophila virilis Proc Natl Acad Sci USA 2005, 102:15965-15970.
96. Brookfield J: Models of repression of transposition in P-M hybrid dysgenesis by $P$ cytotype and by zygotically encoded repressor proteins. Genetics 1991, 128:471-486.

97. Lewis AP, Brookfield JFY: Movement of Drosophila melanogaster transposable elements other than P elements in a P-M hybrid dysgenic cross. Mol Gen Genet 1987, 208:506-510.

98. Malinska H, Tate J, Matyasek R, Leitch A, Soltis D, Soltis P, Kovarik A: Similar patterns of rDNA evolution in synthetic and recently formed natural populations of Tragopogon (Asteraceae) allotetraploids. BMC Evol Biol 2010, 10:291.

99. Kotseruba V, Pistrick K, Blattner FR, Kumke K, Weiss O, Rutten T, Fuchs J, Endo T, Nasuda S, Ghukasyan A, Houben A: The evolution of the hexaploid grass Zingeria kochii (Mez) Tzvel. $(2 n=12)$ was accompanied by complex hybridization and uniparental loss of ribosomal DNA. Mol Phylo Evol 2010, 56:146-155.

100. Eickbush TH, Eickbush DG: Finely orchestrated movements: evolution of the ribosomal RNA genes. Genetics 2007, 175:477-485.

101. Glass SK, Moszczynska A, Crease TJ: The effect of transposon Pokey insertions on sequence variation in the 28S rRNA gene of Daphnia pulex. Genome 2008, 51:988-1000.

102. Zhang X, Eickbush MT, Eickbush TH: Role of recombination in the long-term retention of transposable elements in rRNA gene loci. Genetics 2008, 180:1617-1626.

103. Castro JP, Carareto CMA: Drosophila melanogaster transposable elements: mechanisms of transposition and regulation. Genetica 2004, 121:107-118.

104. Baack EJ, Whitney KD, Rieseberg LH: Hybridization and genome size evolution: timing and magnitude of nuclear DNA content increases in Helianthus homoploid hybrid species. New Phytol 2005, 167:623-630.

doi:10.1186/1759-8753-5-1

Cite this article as: Vergilino et al: Impact of ploidy level on the distribution of Pokey element insertions in the Daphnia pulex complex. Mobile DNA 2014 5:1.

\section{Submit your next manuscript to BioMed Central and take full advantage of:}

- Convenient online submission

- Thorough peer review

- No space constraints or color figure charges

- Immediate publication on acceptance

- Inclusion in PubMed, CAS, Scopus and Google Scholar

- Research which is freely available for redistribution

Submit your manuscript at www.biomedcentral.com/submit
C Biomed Central 